Applied Mathematical Sciences, Vol. 7, 2013, no. 13, 609 - 614

\title{
The Swap Common Superstring Problem
}

\author{
Anna Gorbenko \\ Department of Intelligent Systems and Robotics \\ Ural Federal University \\ 620083 Ekaterinburg, Russia \\ gorbenko.ann@gmail.com \\ Vladimir Popov \\ Department of Intelligent Systems and Robotics \\ Ural Federal University \\ 620083 Ekaterinburg, Russia \\ Vladimir.Popov@usu.ru
}

\begin{abstract}
In this paper we consider an approach to solve the swap common superstring problem. This approach is based on an explicit reduction from the problem to the satisfiability problem.
\end{abstract}

Keywords: swap common superstring, NP-complete, satisfiability

The algorithmic aspects of different problems of finding regularities are thoroughly studied in theoretical computer science (see e.g. [1] - [15]). In particular, the swap common superstring problem was proposed in [16].

Let

$$
\Sigma=\left\{a_{1}, \ldots, a_{m}\right\}
$$

be a finite alphabet. Let

$$
\mathcal{S}=\left\{S_{1}, \ldots, S_{n}\right\}
$$

be a collection of strings over $\Sigma$. For simplicity, we use $S[i]$ to denote the $i$ th letter in string $S$, and $S[i, j]$ to denote the substring of $S$ consisting of the $i$ th letter through the $j$ th letter. The length of a string $S$ is the number of letters in it and is denoted as $|S|$. Let

$$
\# \text { occ }(X, Y)=|\{i \mid X=Y[i, j]\}| \text {. }
$$


The decision version of the swap common superstring problem can be formulated as following.

THE SWAP COMMON SUPERSTRING PROBLEM (SWCS):

INSTANCE: A collection $\mathcal{S}$ of strings over $\Sigma$, a string $T$, and a positive integer $k$.

QUESTION: Is there a string $S$ such that

$$
\begin{aligned}
|S| & =|T|, \\
\{S[i], S[i+1]\} & =\{T[i], T[i+1]\},
\end{aligned}
$$

for all $1 \leq i<|S|$, and

$$
\left|\left\{i \mid \# \operatorname{occ}\left(S_{i}, S\right) \geq 1\right\}\right| \geq k ?
$$

The problem SWCS is NP-hard [16]. Encoding different hard problems as instances of SAT and solving them with efficient satisfiability algorithms has caused considerable interest (see e.g. [17] - [36]). In this paper, we consider an approach to solve the SWCS problem. Our approach is based on an explicit reduction from the problem to the satisfiability problem.

Let

$$
\begin{aligned}
& \varphi[1]=\wedge_{1 \leq i \leq|T|} \vee_{1 \leq j \leq m} x[i, j],
\end{aligned}
$$

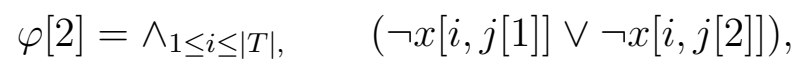

$$
\begin{aligned}
& 1 \leq j[1]<j[2] \leq m
\end{aligned}
$$

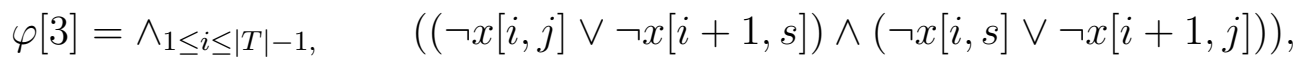

$$
\begin{aligned}
& 1 \leq j \leq m, T[i] \neq a_{j}, \\
& 1 \leq s \leq m, T[i+1] \neq a_{s} \\
& \varphi[4]=\wedge_{1 \leq i \leq k} \vee_{1 \leq j \leq n} y[i, j], \\
& \varphi[5]=\wedge_{1 \leq i \leq k,} \quad(\neg y[i, j[1]] \vee \neg y[i, j[2]]), \\
& 1 \leq j[1]<j[2] \leq n \\
& \varphi[6]=\wedge_{1 \leq i \leq k,}\left(\neg y[i, j] \vee\left(\vee_{1 \leq p \leq|T|-\left|S_{j}\right|+1} z[j, p]\right)\right), \\
& 1 \leq j \leq n \\
& \varphi[7]=\wedge_{1 \leq i \leq k, \quad} \quad(\neg y[i, j] \vee \neg z[j, p[1]] \vee z[j, p[2]]), \\
& 1 \leq j \leq n, \\
& 1 \leq p[1]<p[2] \leq|T|-\left|S_{j}\right|+1 \\
& \varphi[8]=\wedge_{1 \leq i \leq k,}, \quad(\neg y[i, j] \vee \neg z[j, p] \vee \neg x[q, r]), \\
& 1 \leq j \leq n, \\
& 1 \leq p \leq|T|-\left|S_{j}\right|+1, \\
& p \leq q \leq p+\left|S_{j}\right|-1 \text {, } \\
& 1 \leq r \leq m \text {, } \\
& S_{j}[q-p+1] \neq a_{r}
\end{aligned}
$$




$$
\xi=\wedge_{i=1}^{8} \varphi[i] .
$$

It is easy to check that there is a string $S$ such that

$$
\begin{aligned}
|S| & =|T|, \\
\{S[i], S[i+1]\} & =\{T[i], T[i+1]\},
\end{aligned}
$$

for all $1 \leq i<|S|$, and

$$
\left|\left\{i \mid \# \operatorname{occ}\left(S_{i}, S\right) \geq 1\right\}\right| \geq k
$$

if and only if $\xi$ is satisfiable. It is clear that $\xi$ is a CNF. So, $\xi$ gives us an explicit reduction from SWCS to SAT. Now, using standard transformations (see e.g. [37]) we can obtain an explicit transformation $\xi$ into $\zeta$ such that $\xi \Leftrightarrow \zeta$ and $\zeta$ is a 3 -CNF. Clearly, $\zeta$ gives us an explicit reduction from SWCS to $3 \mathrm{SAT}$.

We have designed a generator of natural instances for SWCS. We have considered our genetic algorithms OA[1] (see [38]) and OA[2] (see [39]) for SAT. For solution of SWCS, we have used heterogeneous cluster. Each test was runned on a cluster of at least 100 nodes. Selected experimental results are given in Table 1.

\begin{tabular}{|l|lll|}
\hline time & average & $\max$ & best \\
\hline $\mathrm{OA}[1]$ & $1.22 \mathrm{~h}$ & $11.41 \mathrm{~h}$ & $3.17 \mathrm{~min}$ \\
$\mathrm{OA}[2]$ & $39 \mathrm{~min}$ & $6.23 \mathrm{~h}$ & $6.54 \mathrm{~min}$ \\
\hline
\end{tabular}

Table 1: Experimental results for SWCS.

ACKNOWLEDGEMENTS. The work was partially supported by Analytical Departmental Program "Developing the scientific potential of high school" 8.1616.2011.

\section{References}

[1] V. Yu. Popov, Computational complexity of problems related to DNA sequencing by hybridization, Doklady Mathematics, 72 (2005), 642-644.

[2] V. Popov, The approximate period problem for DNA alphabet, Theoretical Computer Science, 304 (2003), 443-447.

[3] V. Popov, The Approximate Period Problem, IAENG International Journal of Computer Science, 36 (2009), 268-274. 
[4] V. Popov, Approximate Periods of Strings for Absolute Distances, Applied Mathematical Sciences, 6 (2012), 6713-6717.

[5] V. Popov, Multiple genome rearrangement by swaps and by element duplications, Theoretical Computer Science, 385 (2007), 115-126.

[6] V. Popov, Sorting by prefix reversals, IAENG International Journal of Applied Mathematics, 40 (2010), 247-250.

[7] A. Gorbenko and V. Popov, Robot Self-Awareness: Occam's Razor for Fluents, International Journal of Mathematical Analysis, 6 (2012), 14531455 .

[8] A. Gorbenko and V. Popov, The Force Law Design of Artificial Physics Optimization for Robot Anticipation of Motion, Advanced Studies in Theoretical Physics, 6 (2012), 625-628.

[9] A. Gorbenko, V. Popov, and A. Sheka, Robot Self-Awareness: Exploration of Internal States, Applied Mathematical Sciences, 6 (2012), 675688.

[10] A. Gorbenko, V. Popov, and A. Sheka, Robot Self-Awareness: Temporal Relation Based Data Mining, Engineering Letters, 19 (2011), 169-178.

[11] A. Gorbenko and V. Popov, Anticipation in Simple Robot Navigation and Finding Regularities, Applied Mathematical Sciences, 6 (2012), 6577-6581.

[12] A. Gorbenko and V. Popov, Robot Self-Awareness: Formulation of Hypotheses Based on the Discovered Regularities, Applied Mathematical Sciences, 6 (2012), 6583-6585.

[13] A. Gorbenko and V. Popov, Robot Self-Awareness: Usage of Co-training for Distance Functions for Sequences of Images, Advanced Studies in Theoretical Physics, 6 (2012), 1243-1246.

[14] A. Gorbenko and V. Popov, Robot's Actions and Automatic Generation of Distance Functions for Sequences of Images, Advanced Studies in Theoretical Physics, 6 (2012), 1247-1251.

[15] A. Gorbenko and V. Popov, Anticipation in Simple Robot Navigation and Learning of Effects of Robot's Actions and Changes of the Environment, International Journal of Mathematical Analysis, 6 (2012), 2747-2751.

[16] Z. Gotthilf, M. Lewenstein and A. Popa, On Shortest Common Superstring and Swap Permutations, Lecture Notes in Computer Science, 6393 (2010), 270-278. 
[17] A. Gorbenko and V. Popov, On the Problem of Sensor Placement, Advanced Studies in Theoretical Physics, 6 (2012), 1117-1120.

[18] A. Gorbenko and V. Popov, On the Longest Common Subsequence Problem, Applied Mathematical Sciences, 6 (2012), 5781-5787.

[19] A. Gorbenko and V. Popov, Computational Experiments for the Problem of Selection of a Minimal Set of Visual Landmarks, Applied Mathematical Sciences, 6 (2012), 5775-5780.

[20] A. Gorbenko and V. Popov, The Binary Paint Shop Problem, Applied Mathematical Sciences, 6 (2012), 4733-4735.

[21] A. Gorbenko, M. Mornev, V. Popov, and A. Sheka, The Problem of Sensor Placement, Advanced Studies in Theoretical Physics, 6 (2012), 965-967.

[22] A. Gorbenko, V. Popov, and A. Sheka, Localization on Discrete Grid Graphs, Lecture Notes in Electrical Engineering, 107 (2012), 971-978.

[23] A. Gorbenko and V. Popov, The Problem of Selection of a Minimal Set of Visual Landmarks, Applied Mathematical Sciences, 6 (2012), 4729-4732.

[24] A. Gorbenko and V. Popov, The Longest Common Parameterized Subsequence Problem, Applied Mathematical Sciences, 6 (2012), 2851-2855.

[25] A. Gorbenko and V. Popov, Programming for Modular Reconfigurable Robots, Programming and Computer Software, 38 (2012), 13-23.

[26] A. Gorbenko and V. Popov, On the Problem of Placement of Visual Landmarks, Applied Mathematical Sciences, 6 (2012), 689-696.

[27] A. Gorbenko, M. Mornev, V. Popov, and A. Sheka, The problem of sensor placement for triangulation-based localisation, International Journal of Automation and Control, 5 (2011), 245-253.

[28] A. Gorbenko, M. Mornev, and V. Popov, Planning a Typical Working Day for Indoor Service Robots, IAENG International Journal of Computer Science, 38 (2011), 176-182.

[29] A. Gorbenko and V. Popov, SAT Solvers for the Problem of Sensor Placement, Advanced Studies in Theoretical Physics, 6 (2012), 1235-1238.

[30] A. Gorbenko and V. Popov, Clustering Algorithm in Mobile Ad Hoc Networks, Advanced Studies in Theoretical Physics, 6 (2012), 1239-1242.

[31] A. Gorbenko and V. Popov, The Problem of Finding Two Edge-Disjoint Hamiltonian Cycles, Applied Mathematical Sciences, 6 (2012), 6563-6566. 
[32] A. Gorbenko and V. Popov, Hamiltonian Alternating Cycles with Fixed Number of Color Appearances, Applied Mathematical Sciences, 6 (2012), 6729-6731.

[33] A. Gorbenko and V. Popov, Footstep Planning for Humanoid Robots, Applied Mathematical Sciences, 6 (2012), 6567-6571.

[34] A. Gorbenko and V. Popov, Multiple Occurrences Shortest Common Superstring Problem, Applied Mathematical Sciences, 6 (2012), 6573-6576.

[35] A. Gorbenko and V. Popov, The Far From Most String Problem, Applied Mathematical Sciences, 6 (2012), 6719-6724.

[36] A. Gorbenko and V. Popov, Multi-agent Path Planning, Applied Mathematical Sciences, 6 (2012), 6733-6737.

[37] A. Gorbenko and V. Popov, The c-Fragment Longest Arc-Preserving Common Subsequence Problem, IAENG International Journal of Computer Science, 39 (2012), 231-238.

[38] A. Gorbenko and V. Popov, The set of parameterized k-covers problem, Theoretical Computer Science, 423 (2012), 19-24.

[39] A. Gorbenko and V. Popov, Task-resource Scheduling Problem, International Journal of Automation and Computing, 9 (2012), 429-441.

\section{Received: November 1, 2012}

